\title{
Status of development of technology for wireless data transfer in future tracking detectors
}

\author{
Richard Brenner*i \\ Uppsala University, Dept. of Physics and Astronomy, Sweden \\ E-mail: richard.brenner@physics.uu.se
}

The rapid development of high rate and short distance wireless data communication for the consumer market has made the technology capable of meeting requirements of short distance data transfer in future trackers. The limited capacity of data links is currently the bottle neck in trackers which prevents full tracking information to be used for selecting events for further data analysis. A way to increase data transfer capacity without adding hardware services in the tracker is to use wireless links. Although the technology looks promising, technology has to be customised and validated for use in particle physics experiments. I will here discuss the data challenge in future trackers and describe the features that make emerging wireless technology suitable for trackers. I will review the feasibility studies and development done by the WADAPT (Wireless Allowing Data And Power Transmission) project.

The 25th International workshop on vertex detectors

September 26-30, 2016

La Biodola, Isola d'Elba, ITALY

* Speaker.

${ }^{\dagger}$ A footnote may follow. 


\section{Introduction}

Wireless data communication has revolutionized our daily life. Mobile phones, tablets and laptops rely on wireless communication and in the future an increasing number of devices will be connected with radio links to a giant network known as the Internet of Things. For local and short range communication such as Bluetooth and wireless local area networks (WLAN) the unlicensed $2.4 \mathrm{GHz}$ and $5 \mathrm{GHz}$ frequency band are used with maximum data transfer capacity of 2 Mbps for Bluetooth and $600 \mathrm{Mbps}$ for WLAN. For highly portable devices the 3G telecommunication network is used. Depending on country and network capacity, licensed frequencies $850 / 900 / 1700 / 1900 / 2100 \mathrm{MHz}$ are used offering a maximum capacity of $42 \mathrm{Mbps}$. None of these standards meet the requirements for data transfer in tracking detectors where bandwidth of the order of 1 Gbps is required for a single detector module.

In recent years the $60 \mathrm{GHz}$ frequency band has been made available for wireless data transfer offering up to $9 \mathrm{GHz}$ of unlicensed bandwidth. This enables multi-gigabit data transfer capacity for wireless devices. Electromagnetic waves at $60 \mathrm{GHz}$ are, however, absorbed by oxygen and rain which makes the frequency band only suitable for short-range indoor applications. The radiation does not penetrate walls or other obscuring obstacles which makes data communication difficult with moving devices. This might be a problem for users accustomed to mobility but not an issue in trackers. The main commercial application for the technology is short range point-to-point transfer of large media such as uncompressed HD films [2].

The high bandwidth and small feature size make the technology suitable for use in future particle physics experiments. A project called WADAPT [1] (Wireless Allowing Data And Power Transmission) was started by a few groups interested in technology for use in trackers. In chapter 2, I will discuss the data challenges in trackers at the Large Hadron Collider (LHC) at CERN. Chapter 3 will discuss the features that makes the $60 \mathrm{GHz}$ technology suitable for use in trackers. Chapter 4 will review the status of studies performed by WADAPT and ongoing developments of wireless technology for trackers. A short summary and outlook follows in chapter 5.

\section{Data challenges in trackers}

Modern trackers in particle physics must be capable of precise tracking at high particle rates. In order to determine track parameters with sufficient accuracy and to cope with the high particle density at LHC the tracker must measure the position of the traversing particle with about $25 \mu \mathrm{m}$ accuracy. This results in a large number of readout channels with high signal rate which requires large readout bandwitdth. A good example is the recent Insertable B-Layer (IBL) in ATLAS. It has a surface area of $0.15 \mathrm{~m}^{2}$ split into 6 million pixel channels $50 \mu m \times 250 \mu \mathrm{m}$ in size. The data is read out with a combination of low voltage differential wire links and optical links. The IBL is capable of handling hit occupancy of $400 \mathrm{MHz} / \mathrm{cm}^{2}$ with minimal loss of efficiency by a massively paralleled readout using 224 links running at $160 \mathrm{Mbit} / \mathrm{s}$.

It is currently not possible with the technology used today to read out all data from the tracker because of the large mass and volume of services. In the ATLAS detector, full tracking information can only be brought off-detector for 1 event in 400 which affects the physics program. To read out a larger fraction of the events would require more readout links and/or higher readout speed. This 
must be weighted against material mass and power consumption. An important figure of merit for a tracking detector is the radiation length. The smaller the better. Figure 1 shows the simulated radiation length for the CMS tracker split between various contributions. The plot illustrates well a typical detector. Material from cables and cooling dominates over sensors (Sensitive) and electronics in the $\eta$ region between 1 and 2 . The reason is that cables and cooling pipes are routed through this region.

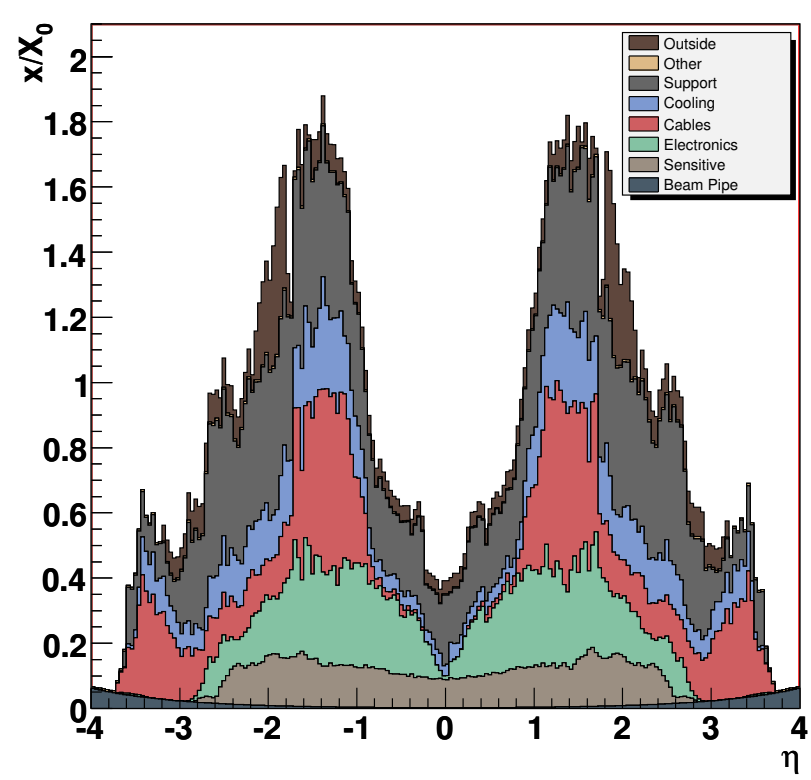

Figure 1: Radiation length distribution in CMS tracker split into main components.

The development of new trackers for the planned high luminosity upgrade of the LHC (HLLHC) is ongoing. At the HL-LHC the luminosity will be ten times higher than the original design value of LHC. The increased luminosity will make tracking more difficult because of higher hit occupancy and larger number of simultaneous proton-proton collisions in each recorded event. At the same time the experiments have to increase the readout rate by an order of magnitude to take advantage of the increase in luminosity. This requires more data links with higher capacity. Wireless transmission would be an attractive technology for low mass data links.

\section{Wireless data transfer in tracking detectors}

A wireless data link requires special transmitter and receiver circuits to transmit data using a high radio frequency carrier. In the transmitter the data at low frequency (baseband) is mixed with a high frequency carrier, generated by a Local Oscillator (LO). The mixed signal is amplified with a power amplifier (PA) before it is transmitted using an antenna. In the receiver the radio signal is received by an antenna, amplified with a low noise amplifier (LNA), down-converted in a mixer using a second LO and demodulated to retrieve the original data. A simplified transceiver architecture is illustrated in figure 2. The baseband is sometimes converted into an Intermediate Frequency (IF), higher than the baseband but significantly lower than that of the carrier. The advantage of an 
intermediate step is that this allows pulse processing using digital modulation methods at a moderate frequency to optimize the usage of bandwidth. In the simplest implementations of a radio link the IF is skipped and the carrier is directly modulated by the baseband. This is called on-off keying (OOK) and has the advantages of simplicity and low power consumption. The disadvantages are the inefficient use of bandwidth and the presence of large side lobes around the main lobe in the frequency spectrum which may cause interference between closely spaced links. By processing the IF signal the problem of the side lobes and use of bandwidth can be improved. A variety of modulation techniques where the frequency, phase and the amplitude of the IF is shifted can be selected. The more advanced modulation methods enable the transmission of several data bits per clock cycle. The drawback is that this requires advanced electronics that increases the power consumption of the link. The most problematic components are DACs and ADCs running at GHz speed. For low power application, as required in trackers, only the simplest modulation techniques such as OOK, binary phase-shift keying (BPSK) and quadrature phase-shift keying (QPSK) can be considered.

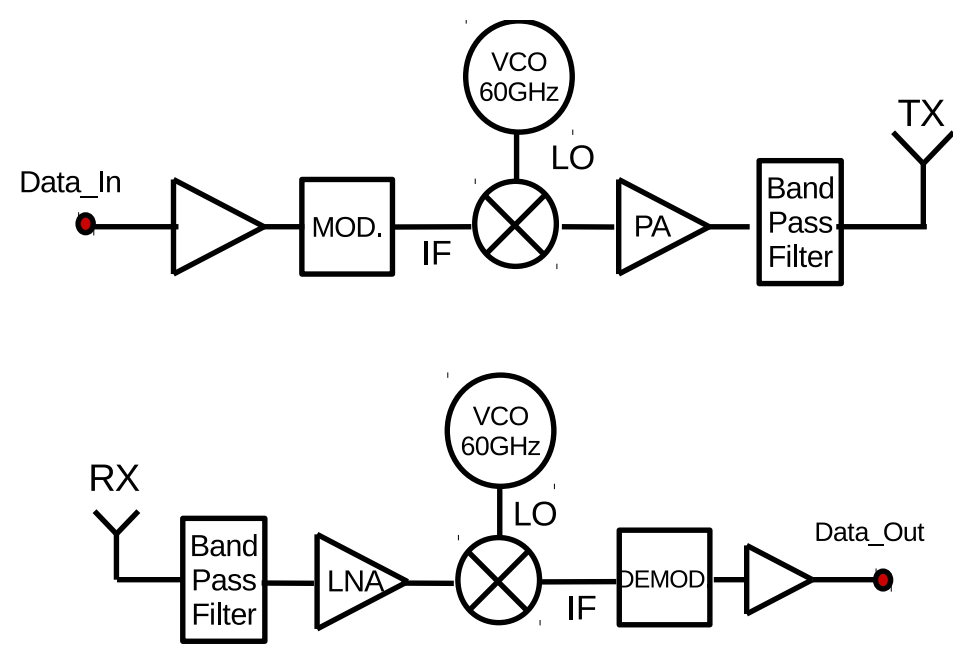

Figure 2: A simplified transceiver architecture for a transmitter (top) and receiver (bottom).

To be used in a future tracker a transceiver must be small in size, have a low power consumption and be radiation hard. High carrier frequency leads to small components and low power. The wavelength of $60 \mathrm{GHz}$ is just $5 \mathrm{~mm}$ and for antennas made of materials with higher permittivity than air the length will be even shorter. The relative permittivity of materials used for microstrip antennas suitable for use in a tracker is around 3 which leads to a dipole antenna size of $2.9 \mathrm{~mm}$. The high relative permittivity of silicon $\left(\varepsilon_{r}=11.7-11.9\right)$ might be considered as an advantage for further size reduction and for improved integration of the antenna with the transceiver electronics. This would however not give an efficient antenna because of large heat losses in the material caused by the presence of the electric field in silicon. This effect can be mitigated by adding layers of polymer, metals and oxide on top of the circuit and by removing silicon material from the backside by etching, forming antenna bridges and beams suspended in air. Even if the most compact link is achieved by integrating the transceiver with the antenna, the most practical solution for fast tests and prototyping is to build a package with the transceiver connected to a separate antenna. 
Previously, high speed electronics suitable for RF applications were fabricated in SiGe BiCMOS and GaAs HBT processes. The shrinking of analogue CMOS processes has made it possible to fabricate high speed transistors. This opens for the fabrication of $60 \mathrm{GHz}$ transceivers in CMOS. The circuits will have low power consumption and can be made radiation hard. A big advantage is the possibility to integrate all functions required to collect and transmit data on the detector module in a single chip. Figure 3 (left) shows the TX-energy per transmitted bit as function of output power using mm-wave transceivers fabricated in CMOS , GaAs and SiGe technologies. The transmitted data range and required energy for a wireless link depend on the combined performance of transceiver with antenna. The transceiver developments are targeting different applications from extremely short distance chip-to-chip intercommunication to short distance transfer of video and medium distance transfer of large data volumes. An example of a integrated wireless CMOS transceiver running at $60 \mathrm{GHz}$ that could meet the requirements of HEP experiments is produced in $90 \mathrm{~nm}$ technology[3]. It has been reported that it can transfer data at 4Gbps rate with a bit error rate $<10^{-11}$ over $1 \mathrm{~m}$ distance using only $170 \mathrm{~mW}$ power. This gives a energy per transmitted bit around $40 \mathrm{pJ} / \mathrm{bit}$. For comparison, Figure 3 (right) shows the reported TX-energy per transmitted bit as function of link distance for a number of CMOS, GaAs and SiGe transceivers.
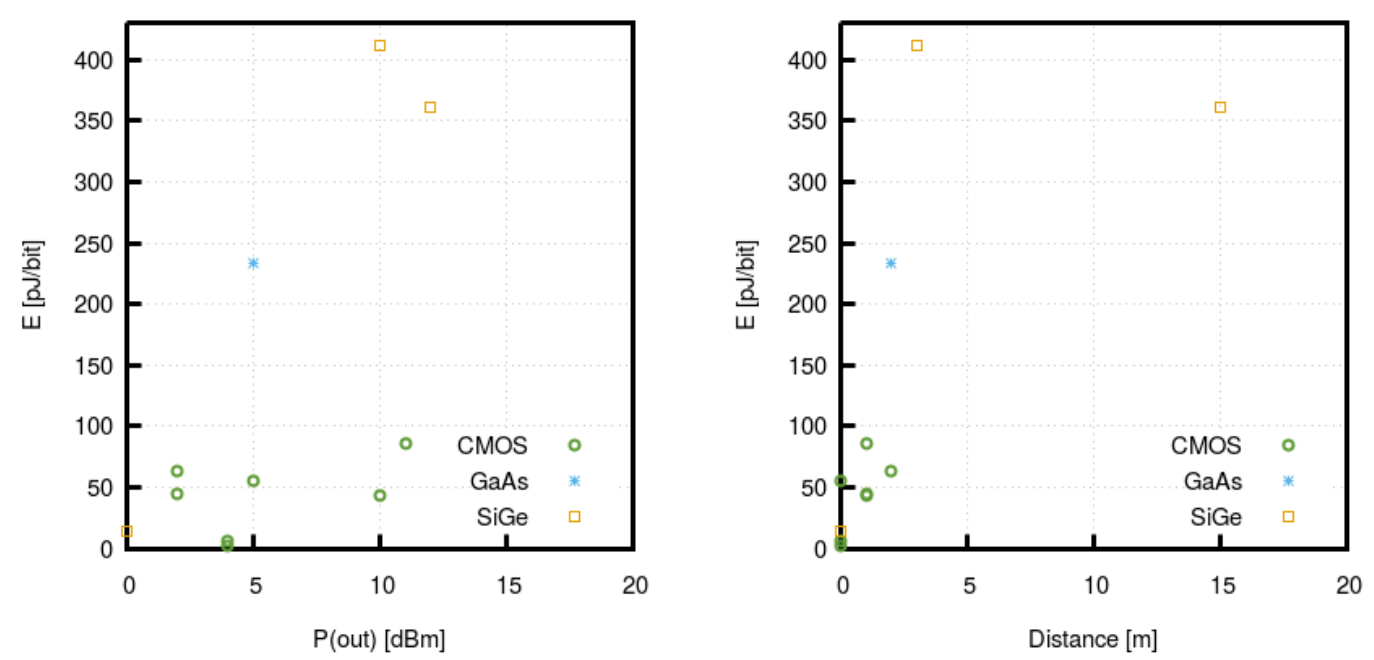

Figure 3: Reported energy per transmitted bit as function of output power of transceiver (left) and distance between TX and RX (right) for transceivers produced in CMOS, GaAs and SiGe [3],[9],[10],[11],[12],[13],[14],[15],[16],[17],[18].

\section{Studies of $60 \mathrm{GHz}$ wireless technology in tracking detectors}

Presently the readout of a detector module is done with a combination of parallel and serial links. The parallel electrical links are used locally on detector module while the serial optical link carries the data from the detector module to the counting room. The wireless link can not replace the optical link entirely since the material between the detector and the counting room prevents this. The wireless link could however ease bringing data from the front-end circuits to a place just outside the tracker where the readout could continue with optical links. This could remove the 
need for parallel buses on the detector module, connectors for readout inside the tracker volume and move the optical transmitter out of the high radiation environment. Wireless links could also facilitate interlayer communication, enabling advanced data reduction to be implemented in the detector reducing the amount of data to be transferred off detector. Several studies have been performed to demonstrate the feasibility of wireless data transfer in trackers. The focus has been on features special for trackers which will not be investigated for commercial applications.

\subsection{Wireless data transfer between tracking layers}

Wireless links are well suited for improving the radiation length in particular in the region that is today densely populated with services. The shortest path is to send data radially out from the detector layers rather than as currently axially along the layers. Each module can send data radially to a receiver positioned on the inside of the solenoid cryostat where it can be converted to optical and transmitted to the outside. In this architecture the material from data links will be distributed evenly over a large surface, hence no accumulation of services as currently in the $\eta$ region between 1 and 2.

A significant problem is however that the metal patterns on sensors, hybrids and ASICs in the tracker make it opaque to RF. A solution investigated is to add an active or passive structure on the detector that carries the signal around/through the layers. Other possible solutions are to beam the signal through gaps in the structure. Figure 4 shows the signal attenuation when transferred through a gap in single layer. The gap is simulated by drilling a circular hole in the layer which is made of metal. The plot shows the attenuation as a function of hole diameter and for two layer thicknesses. The attenuation in a thick layer with hole sizes less than half the carrier wavelength is significantly higher than in the thin layer. By adding a plano-convex dielectric lens, printed in polyethylene with a 3D printer, between the transmitter and the $2.5 \mathrm{~mm}$ hole the attenuation can be reduced significantly for the thick layer (yellow star) while the effect is marginal for the thin layer (red diamond). The blue dotted line shows the behaviour expected from the Bethe-Bouwkamp power law for small holes.

A passive repeater structure consisting of two quad patch antennas connected with a microstrip line has been produced [4]. The repeater was measured to attenuate the signal with $10 d B$ (green dotted line). In the future a $10 d B$ gain low noise amplifier will be added to the repeater structure which will compensate the attenuation through a layer with the drawback of an increase in power.

\subsection{Antenna design and fabrication tolerances}

To build an efficient system the different parts of the system must be precisely matched to prevent loss of efficiency. Patch antennas are easy to fabricate with standard etching techniques on PCB material for high frequency purposes (eg Roger ULTRALAM 3850 or DuPont Pyralux AP9161R). The fabrication tolerances for patch antennas has been studied in order to determine production requirements. The studies show that tolerances better than $5 \mu \mathrm{m}$ is required for these materials to tune the resonant frequency of the antenna with a precision better than $1 \mathrm{GHz}$ [5]. Even higher precision is required for material with higher dielectric constant. 


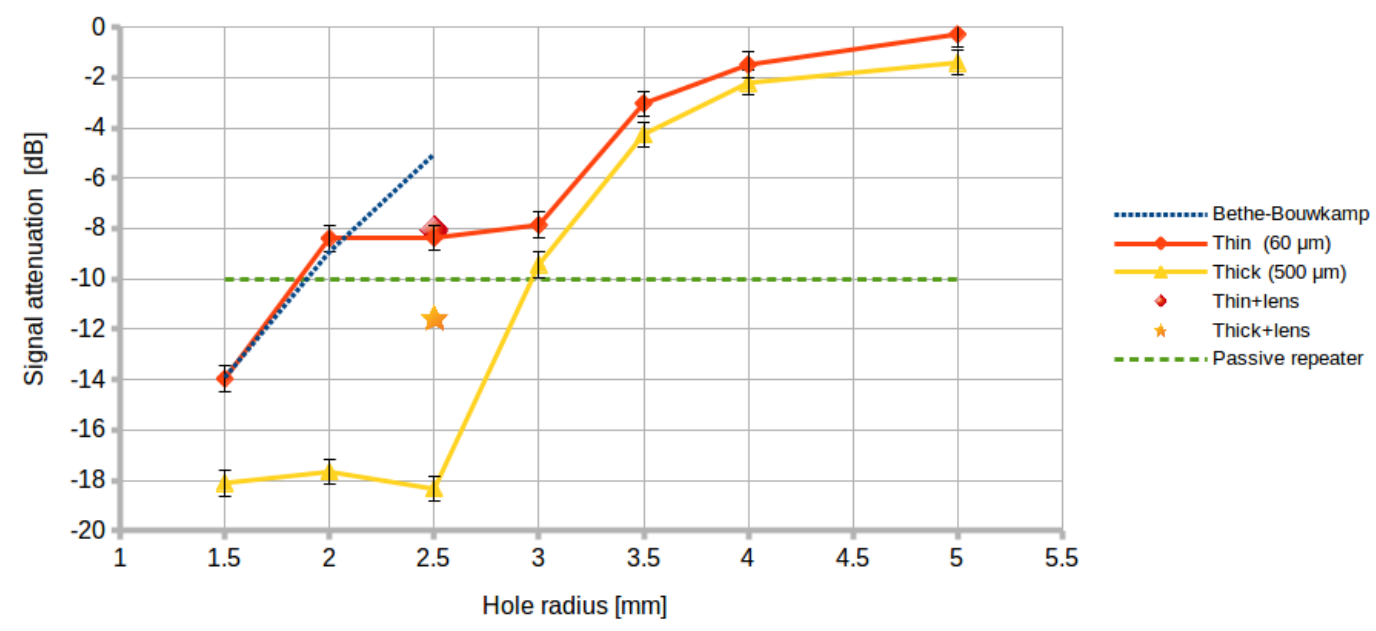

Figure 4: Signal transfer through a single layer

\subsection{Study of link granularity in trackers}

A tracker will require a large number of links placed in a small volume. The baseline is to equip every detector module with a radio transmitter. A typical dimension of a detector module is about $10 \mathrm{~cm} x 10 \mathrm{~cm}$. The size is smaller closer to the interaction point and larger further away. The link granularity supersedes any commercial application. Studies have been performed to investigate how close wireless links can be position before crosstalk becomes a problem and to study methods to mitigate crosstalk. The link pitch measurement was done with open waveguides with poor directivity and waveguides connected to horn antennas giving high directivity. The measurement was done for a TX to RX distance of $10 \mathrm{~cm}$ (compatible with the separation of silicon layers in a tracker). A signal $S / N>20 d B$ was required to ensure low bit error rate. The results from the study [6] show that link pitches down to $5 \mathrm{~cm}$ is feasible. As expected, directive antennas worked significantly better than waveguides but links could be placed as close as $10 \mathrm{~cm}$ even with properly shielded waveguides. The use of polarisation reduces crosstalk even further.

\subsection{Transceiver development for tracking detectors}

The initial tests are done so far with generic test boards too bulky to be used in a tracker. The next step is to either find a transceiver developed for some application that can be qualified for use in trackers or to develop a custom made transceiver for our purposes.

Studies of a compact transceiver designed by CEA LETI [7] for the consumer market is in progress. The circuit is fabricated in CMOS $65 \mathrm{~nm}$ SOI high resistivity technology with an antenna integrated into a single package. Data rates of $2 \mathrm{Gbps}$ over a distance of $7.5 \mathrm{~cm}$ with $100 \mathrm{~mW}$ power consumption have been demonstrated.

A prototype specifically designed for use in a tracker is currently under development at University of Heidelberg [8]. The transceiver will use OOK modulation. Utilizing the full spectral bandwidth the link should be capable of transferring data at about 4 Gbps over a distance of $20 \mathrm{~cm}$ consuming under $150 \mathrm{~mW}$ power. The transceiver is planned to be fabricated in $130 \mathrm{~nm}$ SiGe HBT BiCMOS technology. 


\section{Summary and outlook}

The $60 \mathrm{GHz}$ wireless technology is rapidly developing for high rate, short range data transfer applications. The technology is well suited for use in current and future trackers and could increase the data transfer capacity without adding wires or fibres in the tracker. First feasibility studies have been done with promising outcome. Work is in progress to develop a wireless data link suited for integration on detector modules. It is unlikely that the demonstration of technology will be ready in time for the High Luminosity LHC but one can expect the technology to be used in next generation detectors. With the trends of RF electronics one can expect that the wireless data links will in future move to higher frequencies giving even higher data transfer capacities. The WADAPT project was started to develop wireless technology for use in trackers. The groups behind WADAPT welcome more partners to the project.

\section{References}

[1] E. Locci et al. Development Of Wireless Techniques In Data And Power Transmissson Application For Particle-Physics Detectors, https://arxiv.org/pdf/1511.05807.pdf, (2015)

[2] S.K. Yong, C.-C. Chong, An overview of multigigabit wireless through millimeter wave technology: Potentials and technical challenges, EURASIP Journal on Wireless Communications and Networking, vol. 2007, (2007)

[3] C. Marcu et al.,A 90nm CMOS Low-Power $60 \mathrm{GHz}$ Transceiver with Integrated Baseband Circuitry, In proceedings of International Solid State Circuits Conference - ISSCC 2009, Februaray 8-12, (2009)

[4] D. Pelikan et al., Radial transfer of tracking data with wireless links, PoS (TIPP2014)0095, (2014)

[5] D. Pelikan et al., Wireless data transfer with mm-waves for future tracking detectors, JINST 9, (2014)

[6] S. Dittmeier et al., $60 \mathrm{GHz}$ wireless data transfer for tracker readout systems - first studies and results, JINST 9, (2014)

[7] A. Siligaris et al., A low power 60-GHz 2.2-Gbps UWB transceiver with integrated antennas for short range communications, Conference: Radio Frequency Integrated Circuits Symposium (RFIC), IEEE, (2013)

[8] H. K. Soltveit et al., Multi-Gigabit Wireless data transfer at $60 \mathrm{GHz}$, JINST 7, (2012)

[9] A. Tomkins et al., A zero-IF $60 \mathrm{GHz}$ transceiver in $65 \mathrm{~nm} C M O S$ with . 3.5Gb/s links, Custom Integrated Circuits Conf. (CICC),San Jose, Sept. 200

[10] E. Juntunen et al., A 60-GHz 38-pJ/b 3.5-Gb/s $90 \mathrm{~nm}$ CMOS OOK digital radio, IEEE Trans. Microw. Theory Techn., vol. 58, no. 2, pp. 348âĂŞ355, Feb. 2010.

[11] J. Lee, Y. Huang, Y. Chen, H. Lu, and C. Chang, A low-power fully integrated $60 \mathrm{GHz}$ transceiver system with OOK modulation and on-board antenna assembly, IEEE Int. Solid-State Circuits Conf. Tech. Dig., Feb. 2009

[12] C. W. Byeon, C. H. Yoon, and C. S. Park, A 67-mW 10.7-Gb/s 60-GHz OOK CMOS transceiver for short-range wireless communications, IEEE Trans. Microw. Theory Techn., vol. 61, no. 9, Sep. 2013.

[13] S. Saponara et al.,Design of a $2 \mathrm{~Gb} / \mathrm{s}$ Transceiver at $60 \mathrm{GHz}$ with Integrated Antenna in Bulk CMOS Technology, Proceedings of the 9th European Microwave Integrated Circuits Conference, Rome, Italy, 6-7 Oct. 2014 
[14] S. Gunnarsson et al., $60 \mathrm{GHz}$ Single-Chip Front-End MMICs and Systems for Multi-Gb/s Wireless Communication, IEEE Journal of Solid-State Circuits, Vol. 42, No. 5, May 2007

[15] K. Ohata et al., 1.25Gbps wireless Gigabit Ethernet link at 60GHz-band, 2003 IEEE MTT-S Int. Microwave Symp. Dig., pp. 373-376, June 2003

[16] G. Srdjan et al. Fully integrated $60 \mathrm{GHz}$ transceiver in SiGe BiCMOS, RF modules, and 3.6 Gbit/s OFDM data transmission, International Journal of Microwave and Wireless Technologies, 2011

[17] Reynolds, S. et al., Second generation 60-GHz transceiver chipset supporting multiple modulations at Gb/s data rates, Bipolar/BiCMOS Circuits and Technology Meeting (BCTM), Sept. 2007

[18] W. Chen et al., A 6-Gb/s wireless inter-chip data link using 43-GHz transceiver and bond-wire antennas, IEEE J. Solid-State Circuits, vol. 44, no. 10, Oct. 2009 